\title{
A PROOF OF THE BURKHOLDER THEOREM FOR MARTINGALE TRANSFORMS
}

\author{
T. SHINTANI
}

\begin{abstract}
If $g$ is the transform of an $L^{1}$-bounded martingale $f$ under a predictable sequence $v$ satisfying $\sup _{n}\left|v_{n}\right|<\infty$ almost everywhere, then a proof of the convergence of $g$ is given using an approximation of $f$ by a martingale of bounded variation.
\end{abstract}

Let $(\Omega, A, P)$ be a probability space, and $M^{1}$ the space of $L^{1}$-bounded martingales $f=\left(f_{1}, f_{2}, \ldots\right)$ relative to a fixed increasing sequence $A_{1}, A_{2}, \ldots$ of sub- $\sigma$-fields of $A$. Equipped with the norm $\|f\|_{1}=\sup _{n}\left\|f_{n}\right\|_{1}, M^{1}$ is a Banach space.

A martingale $f$, with $f_{n}=\sum_{k=1}^{n} d_{k}, n>1,\left(d_{k}=f_{k}-f_{k-1}, d_{1}=f_{1}\right)$ is of bounded variation if $\sum_{k=1}^{\infty}\left|d_{k}(\omega)\right|<\infty$ for almost all $\omega$.

Let $B V=\left\{f \in M^{1}: f\right.$ is of bounded variation $\}$. Then, $B V$ is dense in $M^{1}$ in $M^{1}$-norm (Theorem 1 of [3, p. 166]).

The following basic convergence theorem is well known:

TheOREM (THEOREM 1 OF [1]). Let $f=\left(f_{1}, f_{2}, \ldots\right)$ be an $L^{1}$-bounded martingale and let $v=\left(v_{1}, v_{2}, \ldots\right)$ be a predictable sequence of random variables: $v_{k}: \Omega \rightarrow \mathbf{R}$ is $A_{k-1}$-measurable, $k>1$, such that $\sup _{n}\left|v_{n}\right|<\infty$ a.e. Then the martingale transform $g=\left(g_{1}, g_{2}, \ldots\right)$, defined by $g_{n}=\sum_{k=1}^{n} v_{k} d_{k}$, converges a.e.

What is not so transparent is the mechanism of convergence for martingale transforms, i.e., Burkholder transforms. Here is a proof:

Proof. By a result of Burkholder and Shintani (Theorem 1 of [3]), for $f$ in $M^{1}$ and arbitrary $\varepsilon>0$ there is a martingale $f^{(\varepsilon)}$ in $B V$ such that $\left\|f-f^{(\varepsilon)}\right\|_{1}<\varepsilon^{2}$. Let

$$
g_{n}^{(e)}=\sum_{k=1}^{n} v_{k} d_{k}^{(e)}, \quad d_{k}^{(e)}=f_{k}^{(e)}-f_{k-1}^{(e)}, \quad k>1
$$

Then, for almost all $\omega \in \Omega$,

$$
\left|g_{n}^{(e)}(\omega)\right| \leqslant \sum_{k=1}^{n}\left|v_{k}(\omega)\right|\left|d_{k}^{(e)}(\omega)\right| \leqslant \sup _{n}\left|v_{n}(\omega)\right| \cdot \sum_{k=1}^{\infty}\left|d_{k}^{(e)}(\omega)\right|<\infty .
$$

Received by the editors September 1, 1979 and, in revised form, June 23, 1980.

AMS (MOS) subject classifications (1970). Primary 60G45, 46B99.

Key words and phrases. Martingale, almost everywhere convergence, bounded variation, approximation, Burkholder transform, Banach space. 
This means that the sequence $\left\{g_{n}^{(e)}(\omega), n>1\right\}$ converges absolutely for almost all $\omega$. So, $P\left(\lim \sup _{m, n \rightarrow \infty}\left|g_{m}^{(e)}-g_{n}^{(\varepsilon)}\right|>\varepsilon\right)=0$. Then

$$
\begin{aligned}
& P\left(\limsup _{m, n \rightarrow \infty}\left|g_{m}-g_{n}\right|>3 \varepsilon\right) \\
& \leqslant P\left(\limsup _{m, n \rightarrow \infty}\left(\left|g_{m}-g_{m}^{(e)}\right|+\left|g_{n}^{(e)}-g_{n}\right|+\left|g_{m}^{(e)}-g_{n}^{(e)}\right|\right)>3 \varepsilon\right) \\
&< P\left(\limsup _{m, n \rightarrow \infty}\left|g_{m}-g_{m}^{(e)}\right|>\varepsilon\right)+P\left(\limsup _{m, n \rightarrow \infty}\left|g_{n}^{(e)}-g_{n}\right|>\varepsilon\right) \\
&+P\left(\limsup _{m, n \rightarrow \infty}\left|g_{m}^{(e)}-g_{n}^{(e)}\right|>\varepsilon\right) \\
&= 2 \cdot P\left(\inf _{m>1}\left(\sup _{m<n}\left|g_{n}-g_{n}^{(e)}\right|\right)>\varepsilon\right) \\
& \leqslant 2 \cdot P\left(\sup _{n}\left|g_{n}-g_{n}^{(\varepsilon)}\right|>\varepsilon\right) .
\end{aligned}
$$

Now, by the weak $L^{1}$-inequality of Burkholder, for each constant $c>0$ there is a universal constant $C>0$ such that if $|v|<c$ uniformly then

$$
P\left(\sup _{n}\left|g_{n}\right|>\lambda\right)<C \cdot \lambda^{-1} \cdot\|f\|_{1}
$$

for $f \in M^{1}$ and all $\lambda>0$. For a proof, see [2].

Therefore

$$
\begin{aligned}
P\left(\limsup _{m, n \rightarrow \infty}\left|g_{m}-g_{n}\right|>3 \varepsilon\right) & <2 C \cdot \varepsilon^{-1} \cdot\left\|f-f^{(\varepsilon)}\right\|_{1} \\
& <2 C \cdot \varepsilon \text { for all } \varepsilon>0 .
\end{aligned}
$$

Since $\sup _{n}\left|v_{n}\right|<\infty$ a.e., this means that $\left\{g_{n}(\omega), n>1\right\}$ is a Cauchy sequence for almost all $\omega$. Since the state space $X=\mathbf{R}$ is complete, $\lim _{n \rightarrow \infty} g_{n}(\omega)$ exists for almost all $\omega$ and belongs to $X$. This implies that $g$ converges a.e. and the theorem is proved.

\section{REFERENCES}

1. D. L. Burkholder, Martingale transforms, Ann. Math. Statist. 37 (1966), 1494-1504.

2. __ A sharp inequality for martingale transforms, Ann. Probability 7 (1979), 858-863.

3. D. L. Burkholder and T. Shintani, Approximation of $L^{1}$-bounded martingales by martingales of bounded variation, Proc. Amer. Math. Soc. 72 (1978), 166-169.

4. A. M. Garsia, Martingale inequalities: Seminar notes on recent progress, Benjamin, Reading, Mass., 1973.

Department of Mathematics, Tomakoma Technical College, Tomakoma, Hokxaido, Japan 